\title{
PERCEPCIONES DE ESTUDIANTES SOBRE EL PROCESO DE ENSEÑANZA-APRENDIZAJE ASOCIADO AL USO GUIADO DE LA PLATAFORMA TECNOLÓGICA ALEKS EN TIEMPOS DE PANDEMIA
}

\author{
STUDENTS' PERCEPTIONS OF THE TEACHING-LEARNING PROCESS \\ ASSOCIATED WITH THE GUIDED USE OF ALEKS TECHNOLOGICAL \\ PLATFORM IN TIMES OF PANDEMIC
}

Felipe Cristián Marín Álvarez*, Gala Catalina Fernández Frésard**

\section{Resumen}

El presente artículo indaga en el proceso de enseñanza-aprendizaje de la asignatura de Matemáticas en estudiantes universitarias(os) de primer año de la carrera de Ingeniería Comercial, en una universidad privada chilena durante el año académico 2020. El objetivo del estudio fue identificar las percepciones desarrolladas por las(os) estudiantes sobre el proceso de enseñanza-aprendizaje de la asignatura observada, asociadas al trabajo con la plataforma interactiva Aleks, en contexto de pandemia. Los resultados dieron cuenta de percepciones favorables, alta recomendación y una actitud positiva al estudio virtual cuando es acompañado por la plataforma utilizada. El estudio fundamenta la continuidad del uso de Aleks en la asignatura observada y hace posible proyectar una futura ampliación de su implementación en otras asignaturas y carreras.

* Licenciado en Ingeniería Mecánica, Magíster en Docencia para la Educación Superior. Universidad Andrés Bello. email: felipe.marin@unab.cl, ORCID https://orcid.org/00000002-2345-3104

** Actriz, Licenciada en Actuación, Magíster en Artes. Universidad Católica de Chile. email: gala.f@uc.cl, ORCID https://orcid.org/0000-0001-7496-6447 


\title{
Palabras clave:
}

Aprendizaje activo, matemáticas, TIC, estudiante universitario, estrés mental, enseñanza multimedia.

\begin{abstract}
This article investigates the teaching-learning process of the subject Mathematics 1 in first year university students of commercial engineering at a private Chilean university during the academic year 2020. The objective of the study was to identify the perceptions that students had about the teaching-learning process of the observed subject, associated to the work with the interactive platform Aleks, in the context of pandemic. The results showed favorable perceptions, high recommendation, and a positive attitude to virtualized study, when accompanied by the platform used. The study supports the continuity of the use of Aleks in the observed subject and makes it possible to project a future expansion of its implementation in other subjects and careers.
\end{abstract}

\section{Keywords:}

Active learning, mathematics, information and communication technologies (ICT), university student, mental stress, multimedia teaching.

\section{Introducción}

\subsection{Contexto de pandemia}

E1 11 de marzo del 2020, la Organización Mundial de la Salud (OMS, 2020) declaró al brote del virus SARS COV-2 (Covid-19) como una pandemia. Se comunicó al mundo sobre su elevado potencial de propagación, emitiendo alertas con referencia a las consecuencias e impacto en los sectores de salud pública, sociales y económicos.

En aquella fecha, el virus, cuyo origen se localizó en China en diciembre del 2019 (Chen, et al. 2020), ya se había propagado a 114 países en un lapso de 2 semanas, contabilizando más de 118.000 
casos de personas infectadas y 4.291 defunciones (González, Tejada, Espinoza y Ontiveros., 2020). En este contexto, muchos sectores han debido replantear su actividad, cambiar sus estrategias y/o aplicar recursos de innovación, para asegurar su continuidad en situación de confinamiento; uno de esos sectores es la educación universitaria.

En Chile todas las instituciones universitarias han tenido que implementar el uso de plataformas virtuales o salones de videoconferencia para realizar clases y dar continuidad a sus modelos educativos. Cuando un estudiante ingresa a la educación universitaria se encuentra con un continuo de cambios y vivencias, marcados por diferentes factores que van desde las conductas que trae adquiridas en el período escolar; el desarrollo emocional regulado por la relación con sus pares y lazos con la familia -visto como un sistema en constante interacción- hasta un plano actitudinal que presenta frente al estudio (Marín, 2018). Una de las asignaturas en que es posible observar lo anterior, es la de matemáticas, no sólo por el nivel de dificultad de su contenido, sino por las respuestas emocionales que ésta trae consigo para las(os) estudiantes, quienes muchas veces ya traen estas emociones desde la concurrencia de su cotidiano (Vejar y Ávila, 2020).

\subsection{El panorama del ingreso a la universidad}

El impacto que el confinamiento ha tenido en el ámbito de lo psicológico en estudiantes universitarios en cuarentena ha sido reportado por Khan et al. (2020), asociado a vivencias de ansiedad, crisis de pánico, trastornos del sueño, ira y desilusión como manifestaciones frecuentes. En China se realizó un estudio con 1210 universitarias(os), pertenecientes a una zona donde se inició el brote de Covid-19, los resultados mostraron en las(os) jóvenes síntomas como depresión, estrés y ansiedad (Wang, et al., 2020).

En España, Ozamiz, Dosil, Picaza y Idoiagamondragon (2020), reportan que los jóvenes cuyas edades fluctuaban entre 18 y 25 años presentaban niveles más altos de estrés, ansiedad y depresión, en 
comparación con los grupos de personas cuyas edades fluctuaban entre 26 y 60 años; además, González et al. (2020), refieren que el mayor impacto se producía en aquellas personas que presentaban síntomas en enfermedades crónicas.

A partir de la Declaración de Pandemia por el Covid-19 por parte de la Organización Mundial de la Salud (OMS), la Sociedad Interamericana de Psicología (SIP 2020), emitió un mensaje a toda su comunidad para actuar con responsabilidad, empatía, solidaridad y tranquilidad frente a esta nueva situación que se presentaba y que significaba un riesgo de salud pública y comenzó a desarrollar actividades que orientaran el quehacer de los psicólogos y las psicólogas ante la pandemia.

En el reciente Informe del Instituto Internacional de la UNESCO para la Educación Superior en América Latina y el Caribe (OEI-IESALC, 2020), se menciona que el impacto de la pandemia relativo a la salud emocional -previsible a corto, medio y largo plazo para las(os) estudiantes de educación superior-, asociado a la pérdida de contacto social y de las rutinas de socialización, debido al aislamiento por confinamiento (Velázquez, 2020), está dentro de las principales consecuencias de la pandemia en estas(os) estudiantes. Las medidas de distanciamiento social, confinamiento, el miedo al contagio y la suspensión de actividades sociales y recreativas han generado niveles altos de estrés en la población (Barraza, 2020).

\subsection{El primer año de universidad de las(os) estudiantes de Chile}

Es importante tener en cuenta que las(os) adolescentes cuentan con sus propias redes sociales y medios de socialización y comunicación, en los que la información divulgada puede ser errónea (Inter-Agency Standing Committee (IASC, 2020); OMS, 2020), lo que pone de manifiesto la necesidad de habilitar canales de diálogo más reflexivos y críticos especialmente en contextos de emergencia, como el confinamiento, en el que pueden darse condiciones propicias para conectar o reconectar ciertos vínculos con las(os) jóvenes de este 
rango etario que por la propia dinámica de la vida contemporánea se fueron diluyendo (Santrock, 2019). En esta línea, el sentimiento de omnipotencia e invulnerabilidad frente a las amenazas, característico de las(os) adolescentes, puede conducir a resistencias y/o transgresiones de las normativas de asilamiento y confinamiento (Velázquez 2020). Dichas manifestaciones no sólo hay que comprenderlas como características prototípicas de esta edad, sino además en el contexto del cambio en las condiciones habituales de socialización y las consecuentes restricciones que se impongan a aquellas.

En este sentido, el ingreso de miles de estudiantes al primer año de universidad durante el año 2020 estuvo marcado por una condición de adaptabilidad, no sólo ante el nuevo desafío que representa la educación universitaria, sino también, frente a la virtualización del aprendizaje y estudio de gran parte de los contenidos, que hasta el 2019, eran entregados de manera presencial. Si se considera que en los cursos de matemáticas para novatas(os) universitarias(os) puede observarse un posicionamiento emocional y actitudinal de las(os) estudiantes que se relaciona con saberes matemáticos anteriores, y está determinado por características personales (Gómez y Marbán, 2019), resulta relevante que el encuentro con estas asignaturas se dé en el año de ingreso a la educación superior, ya que las emociones se suscitan en el escenario de una nueva experiencia multidimensional, en tanto es, subjetiva, somática y motivacional, y está teñida por factores contextuales y culturales (Hofmann, 2018).

\subsection{La plataforma interactiva Aleks en la enseñanza de las matemáticas}

Como se ha referido, sobre todo en el último año, los contextos pedagógicos han evolucionado y con ellos sus prácticas, las que intentan dar respuesta a los cambios, demandas y necesidades emergentes. La combinación de la sociedad del conocimiento y la sociedad en red, constituyen un nuevo espacio social que posee estructuras e interacciones nuevas y más eficientes (Gurung, 2015); de esta forma, ha nacido un nuevo concepto de alfabetización digital, compuesto 
por los conocimientos previos, las competencias centrales y las actitudes y perspectivas (García y Manso, 2018).

Estos cambios en las prácticas pedagógicas y metodológicas, con principios reinventados desde perspectivas innovadoras, han promovido la reflexión sobre el desarrollo de otros espacios formativos y de creación. En los últimos años, se ha instalado el uso de tecnologías aplicadas a la educación, a partir de las que es posible asociar el componente tecnológico con su impacto en la gestión de la información (Cammaerts, 2017). Las TIC son herramientas tecnológicas digitales que apoyan la comunicación y la información (Sánchez, García, Steffens y Palma, 2019) y poseen diversas características, que van desde la inmaterialidad, interactividad, instantaneidad, innovación, y elevada calidad en imagen y sonido, hasta la digitalización e interconexión (Jin y Cho, 2015). A través del uso de TIC, se dispone de una diversidad de herramientas que permiten a las(os) docentes interactuar con sus estudiantes para fomentar la participación, motivación e interés por el tema tratado, con la finalidad de transmitir el conocimiento de una manera significativa (Torres y Velandia, 2017).

La utilización de la 'realidad aumentada' -tecnología que permite combinar el mundo real con el mundo digital, a través de dispositivos tecnológicos-, favorece el desarrollo de algunas de las categorías taxonómicas para la era digital, como es el caso de la categoría 'crear' (García y Manso, 2018). En esta línea, la actualización en materia de educación, reflejada en ajustes de mallas curriculares y programas de asignaturas, ha promovido la priorización de objetivos y contenidos elementales en ciencias básicas y ha hecho necesaria la implementación de estrategias de nivelación de contenidos fundamentales en matemáticas, apuntando al manejo de esta ciencia como una herramienta transversal en las asignaturas de las especializaciones. Así, diversas instituciones universitarias en Chile han planteado estrategias metodológicas de carácter altamente responsivo que apuntan a trabajar conceptos desde el prisma lúdico, con problemas de retroalimentación. Los cuerpos docentes del área matemática han trabajado estrategias de innovación en 
el aula, principalmente a partir de tres aspectos: concentrando la incorporación de contenidos de nivel superior; estableciendo conexiones intra-matemáticas y extra-matemáticas, incorporando materiales visuales, manipulativos y recursos informáticos mediante el pensamiento crítico (Breda, 2020).

Ejemplo de una plataforma educativa con estas características lo constituye el programa Assessment and Learning in Knowledge Spaces (Aleks). Aleks es un sistema de inteligencia artificial desarrollado por un equipo de investigadores de la Universidad de Nueva York y la Universidad de California, con el apoyo de la National Science Foundation y tiene como objetivo formar un marco básico de comprensión de los conceptos matemáticos (Boykin y Xiao, 2009). La utilización de esta herramienta evalúa en primer lugar el conocimiento de las(os) estudiantes mediante una serie de preguntas introductorias o de revisión, que la plataforma llama 'verificación de conocimientos'. Luego, Aleks elige el tema adecuado para cada estudiante, según el resultado de dicha verificación inicial, junto al grado de dificultad de los contenidos con los que se continuará ejercitando (Maćkowski, Brzoza, Żabka y Spinczyk 2018). Para ello, se enfoca en las brechas del conocimiento individual de las(os) estudiantes, empleando herramientas de evaluación y aprendizaje personalizado que fortalezcan sus habilidades; estrategias de flexibilidad de contenido; gestión de recursos; y acceso ilimitado en línea, dado su nivel de adaptabilidad a cualquier dispositivo tecnológico (Boykin y Xiao, 2009).

Aleks utiliza preguntas adaptativas para determinar con rapidez y precisión exactamente lo que un(a) estudiante sabe y no sabe sobre los contenidos del curso, para luego instruirle sobre los temas hacia los que se inclina a aprender, reevaluando periódicamente las respuestas que estos reportan en las siguientes verificaciones de conocimientos, para asegurar que los contenidos aprendidos efectivamente se retengan. Una característica de esta plataforma es que los cursos de Aleks pueden soportar una amplia gama de contenidos y se caracterizan por evitar preguntas de opción múltiple (Nwaogu, 2012). 


\subsection{Pregunta, objetivos y supuesto de la investigación}

Considerando lo anterior, se estimó necesario indagar en las percepciones que tienen las(os) estudiantes sobre el proceso de enseñanza-aprendizaje en esta nueva realidad mediatizada, para ello se puso el énfasis en el proceso de estudio de la primera asignatura de matemáticas -Matemáticas 1- presente en la carrera de Ingeniería Comercial en una universidad privada chilena, cursada durante el primer semestre del año 2020, en la que se implementó el uso de la plataforma de ejercitación interactiva Aleks, la que fue guiada por el profesor y complementó las clases sincrónicas desarrolladas a través de videoconferencias.

Surge así la pregunta: ¿Cuáles son las percepciones que tienen las(os) estudiantes sobre el proceso de enseñanza-aprendizaje de la asignatura Matemáticas 1, asociadas al trabajo con la plataforma interactiva Aleks en contexto de pandemia? Esta interrogante se fundó en la necesidad de conocer la satisfacción que las(os) estudiantes pudieron o no tener con el uso de esta TIC como recurso metodológico fundamental de la asignatura en estudio, dada la completa virtualización de ésta en las circunstancias de confinamiento.

El objetivo general del estudio fue identificar las percepciones de las(os) estudiantes sobre el proceso de enseñanza-aprendizaje de la asignatura Matemáticas 1, asociadas al trabajo con la plataforma interactiva Aleks. Consecuentemente, los objetivos específicos fueron identificar las percepciones de las(os) estudiantes sobre el proceso de enseñanza-aprendizaje respecto de: el diálogo de las(os) estudiantes con la plataforma; el diálogo de las(os) estudiantes con el profesor; y el diálogo de las(os) estudiantes en el contexto de pandemia.

El supuesto de investigación podría dar cuenta de percepciones favorables y disposición positiva al estudio virtualizado con la plataforma Aleks, dado que es una TIC de ejercitación y respuesta inmediata con recursos lúdicos, características que podrían otorgar una 
percepción de satisfacción y alivio a las(os) estudiantes, respecto de la sensación de estrés, asociada al proceso de enseñanza-aprendizaje, incrementada en el contexto de pandemia.

\section{Metodología}

El enfoque epistemológico de la investigación correspondió al paradigma interpretativo, en tanto se consideró que los objetos de conocimiento son construidos y no registrados pasivamente, y que el principio de esa construcción es el sistema de disposiciones estructuradas y estructurantes que se constituye en la práctica y que está siempre orientado hacia funciones prácticas (Vasilachis, 1992).

El enfoque metodológico del estudio fue el hermenéutico, dado su carácter naturalista e interpretativo, en torno a registros y experiencias vividas, a fin de interpretar los fenómenos en términos de los significados que las personas les otorgan (Fuster, 2019).

La metodología de la investigación fue cualitativa, tomando por objeto de estudio el relato de las(os) estudiantes, con foco en el entendimiento del discurso, desde la perspectiva de las ciencias humanas de Fisher, Laubascher y Brook (2016), y en poder comprender lo observado por medio de significados (Guerrero-Castañeda, Prado, Kempfer y Ojeda, 2017), considerando que la investigación cualitativa se construye en un cuadro holístico y complejo, detallando los puntos de vista de los informantes en un escenario natural (Pérez y Moreno 2019), y que el ser humano se encuentra sumergido en el mundo donde toda vivencia o experiencia en la cual está inmerso no se separa de él, por lo que toda experiencia es propensa a ser estudiada, comprendida e interpretada (Guerrero-Castañeda, Menezes y Prado 2019).

El diseño del estudio fue el fenomenológico, en tanto se enfocó en las experiencias individuales y subjetivas de las(os) participantes (Salgado, 2007), indagando en el significado, estructura y esencia de las experiencias vividas y contextualizando dichas experiencias en 
temporalidad, espacio, corporalidad y contexto relacional. Desde esta perspectiva, la investigación pretende comprender, en un nivel personal, los motivos y creencias que están detrás de las acciones (Quecedo y Castaño, 2002), junto con describir y entender los fenómenos desde la perspectiva construida, basado en el análisis de contenidos y temas específicos (Sampieri, 2018); y está enfocado en la experiencia subjetiva de las(os)estudiantes, es decir: significado, estructura y esencia de una vivencia, respecto de un fenómeno.

Se ha escogido una muestra no probabilística, por conveniencia, compuesta por 23 estudiantes que cursaron la asignatura Matemáticas 1, dado que el foco está puesto en la particularidad de sus vivencias respecto del proceso de enseñanza-aprendizaje y sus percepciones. Por tanto, lo que importa es la riqueza, calidad y profundidad de la información por sobre la generalización, la cantidad y estandarización (Scharager y Reyes, 2001).

El criterio de inclusión consideró a todas(os) las(os) estudiantes que cursaron la asignatura Matemáticas 1, semestre 1 de 2020, de la carrera de Ingeniería Comercial de una universidad privada chilena. El escenario de estudio fue una de las secciones del curso Matemáticas 1. Consecuentemente con el objetivo principal de la investigación de identificar las percepciones de las(os) estudiantes sobre el proceso de enseñanza-aprendizaje de la asignatura Matemáticas 1, asociadas al trabajo con la plataforma interactiva Aleks, se decidió realizar la recogida de datos mediante entrevistas estructuradas individuales, las cuales, dado el contexto de confinamiento, fueron en formato remoto.

Una vez cerrado el proceso de calificación, en la última sesión sincrónica del curso, se invitó a quienes desearan participar voluntariamente del estudio, a asistir a la entrevista estructurada en un horario posterior, extraordinario. La invitación se extendió a todas(os) las(os) alumnas(os) regulares del curso, con una semana de anticipación a la fecha de la videoconferencia. Los(as) 23 estudiantes asistentes, en una videoconferencia grupal previa a la entre- 
vista individual, recibieron información detallada sobre el tipo de estudio que se desarrollaría, indicándoles que podrían abandonar éste en cualquier momento que lo deseasen.

Se les informó que el registro de su participación sería anónimo, identificándoles para efectos de análisis posteriores, con un código alfanumérico aleatorio. Todas(os) las(os) estudiantes accedieron voluntariamente y a cada una(o) se le entregó un link personal de acceso a la entrevista para ser respondida en ese mismo momento.

Para la construcción del instrumento correspondiente se consideraron las siguientes categorías apriorísticas: diálogo con la plataforma; diálogo con el profesor y diálogo con el contexto de pandemia. A partir de éstas, se definieron las preguntas de la entrevista estructurada. Este instrumento fue validado, antes de su aplicación, por un experto coordinador del área académica disciplinar, de acuerdo con la estructura jerárquica administrativa de la institución educacional en la que se desarrolló el estudio.

El corpus correspondió a las respuestas escritas de las entrevistas individuales. A partir de este corpus, se abordó un análisis de contenido semántico, pues corresponde a una técnica destinada a formular, a partir de ciertos datos, inferencias reproducibles y válidas que puedan aplicarse a un contexto (Krippendorff, 1990), y en tanto el foco fue el análisis de los mensajes recogidos en las entrevistas, a fin de inferir conceptualizaciones de la producción de estos mensajes, lo que es coincidente con la perspectiva de Bardin (1991). Además, a partir de conceptos comunes o recurrentes que emergieron del análisis al corpus, se definieron las siguientes categorías emergentes asociadas a las categorías apriorísticas predefinidas: características metodológicas; apego y vinculación; motivación extrínseca; estrés en el estudio en pandemia y optimización y aprovechamiento de recursos.

Finalmente, las categorías apriorísticas y categorías emergentes del estudio aplicadas al análisis del corpus, se muestran en la figura 1. 
Figura 1. Categorías de análisis

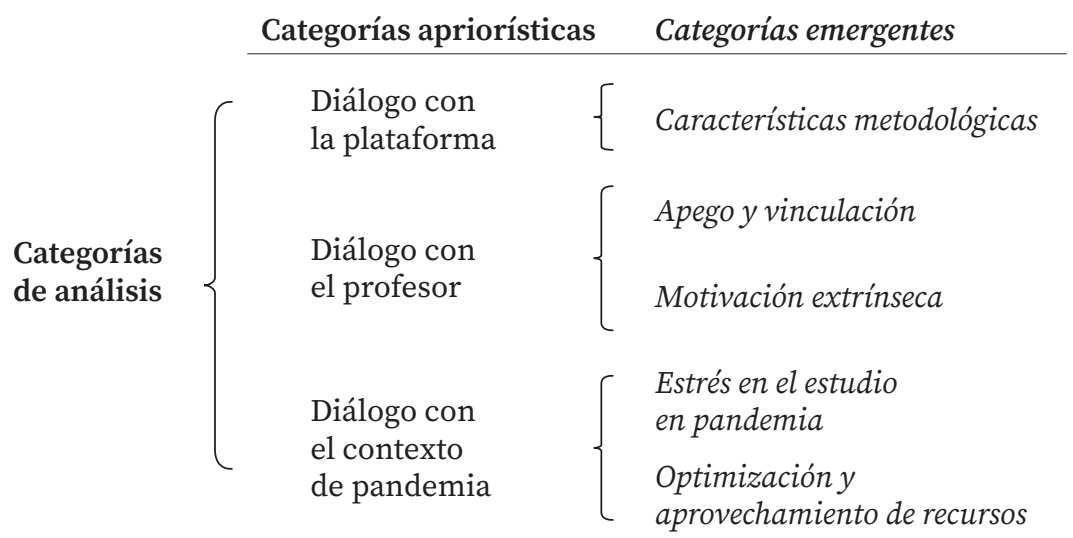

Fuente: Elaboración propia

\section{Resultados}

En la línea de las percepciones que presentan las(os) estudiantes respecto del diálogo con la plataforma, diálogo con el profesor y diálogo con el contexto de pandemia, se observó por separado los significados que cada estudiante construyó, para así tener una imagen global del fenómeno, apuntando a "comprender cómo funcionan todas las partes juntas para formar un todo" (Pérez, 1998, p.81), aspecto que se consideró central, dado que el mundo social está construido de significados y símbolos (Jiménez-Domínguez, 2000) y puede comprenderse, en parte, a raíz de la recepción e interpretación de dichos significados.

De esta manera, las entrevistas realizadas a las(os) estudiantes buscaron un diálogo abierto en el encuentro (Guerrero, et al., 2017) en el cual el fenómeno es transmitido a través del discurso de las personas (Heidegger, 2015). Este discurso es generado por la misma persona, respecto de una vivencia que ha sido experimentada por ella y que ha almacenado en su conciencia, es decir, le ha 
dado significación. De esta forma, lo que se rescata es el discurso ya procesado por la persona (Maurice, 2006).

Teniendo en cuenta estas consideraciones y de acuerdo con el análisis de contenido semántico de las entrevistas, los resultados son los que se presentan a continuación.

\subsection{Categoría apriorística: Diálogo con la plataforma}

Respecto de la categoría apriorística del diálogo con la plataforma, y a partir del análisis de las entrevistas, los datos recogidos hacen referencia a las ventajas y desventajas metodológicas de la plataforma. Como se indicó, se observó una cierta recurrencia o repetición de percepciones en los relatos, lo que provocó el levantamiento de una categoría emergente, llamada características metodológicas, representada por el código $\mathrm{CM}$.

\subsubsection{Categoría emergente: Características metodológicas (CM)}

La mayoría de las(os) estudiantes perciben la plataforma Aleks como excelente, argumentando, por ejemplo: "es una buena plataforma, de mucho apoyo y que permite reforzar contenidos con diversas metodologías" (E2); "trabaja en forma didáctica pues te da premios y puntos por hacer los ejercicios" (E8); "permite trabajar conectado desde mi celular y en cualquier momento" (E16); "antes de hacer un ejercicio, te explica cómo hacerlo, paso a paso" (E20); "si te equivocas, no te deja avanzar y te manda a hacer ejercicios más simples antes de continuar" (E23). Además, se obtuvo que la gran mayoría de las(os) estudiantes consideran que el programa Aleks fue un apoyo para su proceso de enseñanza-aprendizaje y lo recomendarían a sus compañeras(os).

Muchas(os) de las(os) participantes creen que el programa les sirvió en el desarrollo del curso de Matemáticas 1 para poder nivelar contenidos que no recordaban, o bien no habían integrado adecuadamente en sus respectivos establecimientos de educación 
escolar, y consideran un aporte que la plataforma trabaje diversos sets de ejercicios presentados de menor a mayor nivel de dificultad.

Paralelamente, un grupo pequeño de las(os) estudiantes plantean que el sistema de calificación de Aleks es demasiado estricto, pues si los resultados digitados no son idénticos a lo que el programa solicita, se califica con cero puntos, argumentando, por ejemplo, "a pesar de ser un buen programa, tiene algunas cosas que mejorar, por ejemplo, el hecho de que las respuestas sean tan exactas al momento de la evaluación del ejercicio" (E18); como también, "a pesar de que mis respuestas estén bien, pero con algún orden distinto, Aleks lo considera malo y esto hace en ocasiones que mis avances no den frutos y tenga que comenzar nuevamente" (E11). Lo mismo sucede con la calificación de controles, ya que, al terminar un ejercicio, teniendo solo el último paso errado, Aleks considera todo el ejercicio incorrecto. En esta línea, la mayoría de las(os)estudiantes plantearon que trabajar con Aleks consume mucho tiempo, el cual podrían dedicar al estudio y refuerzo de otras asignaturas y, además, "siento que para estudiar online hay que tener voluntad porque es más fácil distraerse” (E5).

Por otro lado, en cuanto a conocimiento y herramientas aportadas por la plataforma y aprendidas en el curso Matemáticas 1, la gran mayoría de las(os) estudiantes indican estar preparados para la siguiente asignatura de matemáticas, gracias al trabajo desarrollado con la plataforma Aleks, argumentando, por ejemplo, "la base que proporciona Aleks es muy buena ya que me ayudó a recordar y reforzar ciertas materias que serían base para después" (E19). Un tema recurrente es la necesidad que expresan las(os) estudiantes de trabajar con un programa como Aleks de manera sostenida, dado que sólo en Matemáticas 1, se trabaja con la plataforma, mientras que los siguientes cursos de matemáticas, pertenecientes a la malla curricular de la carrera en estudio, no tienen apoyo de plataformas virtuales. Respecto de esto, la gran mayoría de las(os) estudiantes considera que se debiera contar con una plataforma de ejercitación durante todos los cursos de matemáticas de la carrera, apuntando 
a que ello constituiría un sello particular y daría un hilo conductor al proceso de formación. Así algunos estudiantes argumentan, por ejemplo, "en el curso de Matemáticas 1, Aleks está todo el tiempo disponible, en cambio en los siguientes cursos, tienes que esperar la clase para hacer consultas; debiera haber plataformas como Aleks en todos los cursos de matemáticas" (E21); "las plataformas de respuesta virtual podrían existir como apoyo constante, como licencia de acceso a los programas mientras dure toda la carrera" (E1).

\subsection{Categoría apriorística: Diálogo con el profesor}

Respecto de la categoría apriorística, diálogo con el profesor, el análisis de las entrevistas da cuenta del rol fundamental del profesor como actor protagónico y activo en el proceso de enseñanza-aprendizaje, cuyo papel, más allá de un mero expositor, es el de motivar, guiar la entrega de contenidos y formar futuros profesionales, características claves del docente, que cobran mayor relevancia en el tiempo de pandemia.

A partir del análisis de contenido semántico realizado se generó el levantamiento de dos categorías emergentes respecto del diálogo con el profesor: la primera referida al apego y vinculación de las(os) estudiantes con el profesor, representada por el código AV; y la segunda referida a la motivación extrínseca que el profesor promueve en las(os) estudiantes, representada por el código ME.

\subsubsection{Categoría emergente: apego y vinculación (AV)}

En consideración a lo anterior, en el marco de la categoría AV, se obtuvo que gran parte de las(os) estudiantes tienen percepciones que muestran al profesor como una figura de apego seguro. Dentro de los principales argumentos entregados se señala, por ejemplo: "siempre nos está brindando muchísimo apoyo, se preocupa por nosotros" (E2); "me hace sentir apoyada por él, lo encuentro bacán" (E9); "tengo una excelente imagen de mi profesor, es comprensivo" (E4); "me dan ganas de ir a sus clases" (E14), "si le pido ayuda, él me 
la da, me responde los correos, me responde en clases, me da consejos" (E17); "el profesor nos ha entendido por lo que estamos pasando" (E22); "me siento muy apoyada ya que el profesor comprende la situación de encierro, estrés y pandemia que hay en estos momentos" (E6).

\subsubsection{Categoría emergente: motivación extrínseca (ME)}

Respecto al análisis de la categoría emergente $\mathrm{ME}$, observada con recurrencia en el análisis de las entrevistas, se obtuvo que las(os) estudiantes tienen percepciones que muestran al profesor como una figura que estimula la motivación, argumentando, por ejemplo: "tiene las habilidades blandas para lograr un proceso de aprendizaje efectivo, genera confianza al momento de querer realizar dudas o consultas"(E12); "mi profe de matemáticas tiene muy buena disposición a la hora de enseñar a sus alumnos" (E15); "me gusta mucho que me despierte el deseo por aprender" (E3); "me siento apoyado y motivado por mi profe de matemáticas" (E5); "nos entregó resúmenes y fórmulas que hacen más fácil el estudio" (E14); "brinda toda la ayuda necesaria para que nos vaya bien en nuestras evaluaciones” (E10).

\subsection{Categoría apriorística: Diálogo con el contexto de pandemia}

Respecto de la categoría apriorística, diálogo con el contexto de pandemia, el análisis de las entrevistas da cuenta de percepciones de agobio, estrés y agotamiento excesivo frente al panorama producido por la emergencia sanitaria. En los relatos de las(os) estudiantes se logra evidenciar la preocupación por el futuro incierto y el miedo a perder la vida de algún familiar cercano, lo que produce un ambiente que dificulta el proceso de enseñanza-aprendizaje. Además, las(os) estudiantes mencionan con bastante recurrencia que el contexto donde se está desarrollando el primer año de estudios universitarios está dado por un constante estrés en el estudio, producido por la pandemia.

Por tanto, a partir del análisis de contenido semántico realizado, se generó el levantamiento de dos categorías emergentes respecto del diálogo con el contexto de pandemia: la primera referida al 
estrés en el estudio en pandemia, representada por el código EP; y la segunda referida a la optimización y aprovechamiento de recursos, representada por el código $\mathrm{OP}$, que hace referencia principalmente a la ventaja de la economía de tiempos y recursos de traslado.

\subsubsection{Categoría emergente: estrés en el estudio en pandemia (EP)}

En consideración con la categoría EP, se obtuvo que la mayoría de las(os) estudiantes tienen percepciones de estrés ante el estudio en contexto de pandemia, argumentando, por ejemplo, "en online se está solo" (E4); "es difícil llevar un estudio online por múltiples razones una de ellas y la que más es frecuente es la caída del internet reiteradamente, es agotador" (E23). En esta línea, argumentaban con frecuencia la percepción de cansancio por el trabajo remoto, por ejemplo, "estar varias horas en el computador es agotador, te arden los ojos, a veces la señal de internet no es buena y no todos los profes entienden esta situación" (E20); "no me gusta porque no tengo toda mi atención a las clases, internet no es estable y mis familiares siempre interrumpen" (E17); "sin tener contacto físico con el profesor y compañeros, no tiene gracia estudiar de esta forma" (E21); "la vista se me ha dañado más, me agota” (E15).

\subsubsection{Categoría: optimización y aprovechamiento de recursos (OP)}

En consideración con la categoría emergente OP, se obtuvo que las(os) estudiantes tienen percepciones de aprovechamiento u optimización de recursos ante el estudio en contexto de pandemia, argumentando, por ejemplo: "no tengo que estar viajando" (E7); "puedo organizar mucho mejor mis tiempos, tengo más espacios para realizar otras actividades" (E11); "cuando se ve el ámbito de tiempos, resulta mejor estudiar en remoto" (E12); "no se pierde tiempo viajando a la universidad lo que da más tiempo para desarrollarse en otros temas" (E18); "salgo de una clase y luego entro a otra, todo desde mi casa y cuando termino mi día de clases, ya estoy en casa, es decir, me evito los traslados" (E19); "ahorro plata en locomoción y tiempo, lo que puedo ocupar en otras cosas” (E20). 


\section{Discusión}

A partir de lo anterior es posible reportar ciertas tensiones producidas al relacionar las percepciones de las(os) estudiantes y los aspectos teóricos expuestos en la introducción, lo cual permite proponer la siguiente discusión: Como se ha mencionado, especialmente en la última década, diversas universidades chilenas han realizado esfuerzos para avanzar en la línea de la incorporación de estrategias metodológicas en la enseñanza de las matemáticas, principalmente con el uso de TIC (Cammaerts, 2017). Desde la incorporación de herramientas o aplicaciones gratuitas en celulares, hasta sistemas más robustos como Aleks, se ha perseguido acercar a las(os) estudiantes hacia el encuentro con las matemáticas; sin embargo, la evolución de los contextos pedagógicos que la implementación de estas herramientas tecnológicas ha traído consigo, determinan un escenario nuevo -el nuevo espacio social que plantea Gurung (2015)al que, tanto profesoras(es), como estudiantes, han debido aprender a adaptarse. Respecto de los resultados obtenidos en el presente estudio, relativos al diálogo que las(os) estudiantes pudieron establecer con la plataforma Aleks, se obtuvo una alta valoración de esta TIC, en tanto entrega retroalimentación inmediata en el desarrollo autónomo de ejercicios los que, además, integran elementos lúdicos. Dicha valoración positiva sería posible a partir de la sensación de acompañamiento sostenido que la plataforma provee con retroalimentación inmediata, característica que disminuiría la sensación de estudio en soledad, tan exacerbada en estudiantes en contextos de confinamiento, y permitiría regular en el proceso de alfabetización digital, el vínculo entre las competencias, actitudes y perspectivas de las(os) estudiantes (García y Manso, 2018) ante el desafío de la ejercitación autónoma.

Además, la inclusión de elementos lúdicos de Aleks, haría el proceso de enseñanza-aprendizaje mucho más llevadero y cercano, a través del establecimiento de conexiones intramatemáticas y extramatemáticas (Breda, 2020), posibilitadas por la combinación del juego y la ejercitación. 
Respecto de los resultados obtenidos relativos al diálogo que las(os) estudiantes pudieron establecer con el profesor como figura de apego, fue posible observar que la imagen que tienen de éste coincide con lo que se considera una figura de apego seguro, lo que se considera de gran relevancia, en el escenario de alta incidencia de variaciones emocionales o desregulaciones generadas por el estrés, la ansiedad y la incertidumbre, producidos por largos períodos de confinamiento (Khan et al., 2020; Wang et al., 2020; Ozamiz et al., 2020), en los que el contacto con compañeras(os) y profesoras(es) se restringe a los espacios de videoconferencias sincrónicas. De acuerdo con la teoría del apego -que se ha abocado al estudio de las relaciones entre adolescentes y adultos y de los procesos psicológicos como funcionamiento interpersonal, emoción y regulación (Mikulincer y Phillip, 2012)-, el profesor como figura de apego seguro para las(os) estudiantes, posibilitaría la baja en la sensación de estrés y en la recurrencia de problemas de salud mental, que se ven alterados en situación de confinamiento. En este sentido, la figura del profesor en el contexto de pandemia habría satisfecho la búsqueda de proximidad con personas significativas en momentos de necesidad (Bowlby, 1988), y habría promovido un sentido de estabilidad en las(os) alumnas(os), contribuyendo a la construcción de representaciones mentales positivas de sí mismos y de los demás (Mikulincer y Phillip, 2012).

Respecto de los resultados obtenidos, relativos a la motivación extrínseca -no generada por el estudiante-que el profesor pudo haber promovido en las(os) estudiantes de primer año universitario, se obtuvieron percepciones de alta motivación por el estudio, generadas por la acción del profesor en el proceso de enseñanza-aprendizaje. Ello se considera de gran relevancia para el buen desarrollo de dicho proceso, dada la relación directa que es posible establecer entre la motivación y el rendimiento académico (Cerasoli, et al., 2014; Cook y Artino, 2016), y las dificultades que el primer año de estudios trae consigo, tanto en el plano relacional y actitudinal (Marín, 2018), como en el emocional (Vejar y Ávila, 2020). Las sensaciones de agobio, cansancio y estrés reportadas por las(os) estudian- 
tes de este estudio -considerablemente aumentadas en contexto de aislamiento por confinamiento (Velázquez, 2020)-, promoverían una necesidad creciente de motivación ante el estudio considerando, además, el escenario desafiante de una nueva experiencia multidimensional en el primer año de ingreso a la universidad (Hofmann, 2018), y las dificultades que este desafío pudiera acarrear. En este sentido, las(os) estudiantes reportaron características y conductas desarrolladas por el profesor que sustentan tanto una motivación por el empleo de técnicas de estudio más rutinarias y memorísticas, que complementarían el aprendizaje con procesos mentales más profundos (Ramírez, et al. 2020); como un apoyo moral, que contribuiría a que las(os) estudiantes se sientan cómodas(os) e identificadas(os) con la universidad, compañeras(os) y docentes, y puedan formar un hábito de estudio y de vida (Quispe, 2017).

En síntesis, los resultados permiten afirmar que, efectivamente, el profesor fue capaz de interactuar con las(os) estudiantes para fomentar su participación, motivación e interés, con la finalidad de transmitir el conocimiento de una manera significativa (Torres y Velandia, 2017).

Respecto de los resultados obtenidos relativos al diálogo de las(os) estudiantes con el contexto de pandemia, la alta valoración de la virtualización del proceso de enseñanza-aprendizaje en contexto de confinamiento, obedecería en gran medida a la optimización de recursos, en especial respecto de la economía en tiempo y costos de traslado y la posibilidad de compatibilizar el estudio con actividades de la vida laboral o personal, lo que deja en evidencia que la carga de responsabilidades que las(os) estudiantes deben enfrentar en un primer año de estudios es excesiva. En este sentido las percepciones que mantienen las(os) estudiantes respecto de una sensación de estrés transversal frente al estudio virtualizado, hace visible que los procesos educativos deben estar bien acompañados en todas las asignaturas que se cursen en paralelo, de manera que las(os) estudiantes puedan mantener un posicionamiento emocional y actitudinal (Gómez y Marbán, 2019) apropiado a la incorpora- 
ción de nuevo conocimiento y acorde a la magnitud de los desafíos de una sociedad en creciente virtualización y cambio.

\section{Conclusiones}

Como resultado de la reflexión sobre las percepciones de las(os) estudiantes del curso matemáticas 1 , sobre el proceso de enseñanza-aprendizaje en esta nueva realidad mediatizada, y el uso de la plataforma interactiva Aleks, guiada por el profesor de la asignatura, se concluye lo siguiente:

Las percepciones que tienen las(os) estudiantes son de valoración altamente positiva asociadas al diálogo tanto con la plataforma, como con el profesor. Asociadas al diálogo con el contexto de pandemia, hay percepciones de valoración negativa en relación al nivel de estrés, y de valoración positiva en relación a la optimización de recursos.

Se observa gran satisfacción hacia el proceso de enseñanza-aprendizaje experimentado por las(os) estudiantes, gracias al refuerzo de contenidos de álgebra elemental que provee Aleks, otorgando una base más sólida para la asignatura. Las características de la plataforma mejor valoradas por las(os) estudiantes son su alto nivel de responsividad (pudiendo ser trabajada en Tablet, iPod, celulares y computadores, en cualquier instante o día de la semana); y la capacidad de trabajo de ejercicios en forma secuenciada (de menor a mayor grado de dificultad).

En general, las percepciones negativas tienen relación con el nivel de exactitud que el sistema exige en la corrección de respuestas (por ejemplo, la forma en que deba estar ordenado un polinomio, o el orden en la que se presenta la escritura de suma de fracciones parciales). Queda expuesta la complejidad del escenario de pandemia y la alta incidencia de indicadores de estrés, devenidos de problemas externos al desarrollo propio del curso tales como dificultad de conectividad y problemas asociados a la disponibilidad y comodidad del espacio físico de estudio. 
Además, los conflictos emocionales relacionados con la falta de contacto físico e interacción presencial, influyen en el nivel de motivación y la continuidad del proceso de enseñanza-aprendizaje, sobre todo considerando que se enfrenta un primer año de estudios.

Por otro lado, es posible concluir que la valoración positiva de la optimización de recursos que las(os)estudiantes reportan, en relación a la mejora considerable en la organización del tiempo y ahorro de dinero, responde a una preexistencia de sobrecarga de actividades, que exige demasiado a las(os)estudiantes, tanto en tiempo, como en dinero. Sin perjuicio de lo anterior, respecto del supuesto del estudio, es posible afirmar que las(os) estudiantes tuvieron percepciones favorables y actitud positiva al estudio virtualizado con la plataforma Aleks, dadas sus características de ejercitación y respuesta inmediata con recursos lúdicos, las que, efectivamente, otorgaron una percepción de satisfacción y alivio a las(os)alumnas/os, respecto de la sensación de estrés asociada al proceso de enseñanza-aprendizaje, incrementada en el contexto de pandemia. Las(os)estudiantes manifiestan el deseo de tener programas como Aleks en los siguientes cursos de matemáticas de la carrera, pues consideran que constituyen un apoyo constante, además del entregado por el docente. Complementariamente, se observa un alto reconocimiento y valoración del apoyo y acompañamiento entregados por el profesor del curso, relevando la flexibilidad metodológica en contexto de pandemia, de lo que es posible concluir, que la implementación de TIC en procesos de enseñanza-aprendizaje, debe necesariamente ser mediada y acompañada por el/la docente, a fin de propiciar una respuesta emocional y actitudinal favorable de las(os) estudiantes ante la herramienta tecnológica, el aprendizaje y el estudio.

\section{Limitaciones}

El presente estudio tuvo como principal limitante, precisamente, el desafío de generar vínculos significativos con las(os) estudiantes en 
el contexto de pandemia. La distancia física hizo muy difícil transferir a las(os) estudiantes la relevancia de emprender el presente proceso de investigación, y de indagar en la evaluación de la implementación de Aleks en la asignatura observada, para motivar su participación en la investigación. Las entrevistas no pudieron ser realizadas en forma presencial, lo que pudo influir en la precisión y detalle con que se recogió el relato de cada sujeto. Además, debido a la sensación de sobrecarga de actividades sincrónicas en pantalla que reportaban las/estudiantes, se optó por la modalidad de entrevista escrita, lo que pudo influir en la espontaneidad de las respuestas reportadas. No obstante, la información recogida fue suficiente para dar respuesta tanto a los objetivos, como a las preguntas y supuestos del estudio.

\section{Proyecciones}

Las proyecciones de esta investigación se relacionan con el beneficio que podría acarrear la implementación de un sistema de apoyo con TIC para todos los cursos de matemáticas, no solo de la carrera de ingeniería comercial, sino también de otros programas de estudio que contemplen matemáticas en sus mallas curriculares. Es posible proyectar el beneficio de la implementación que incorpore el trabajo con TIC en este tipo de asignaturas, puesto que las percepciones que las(os) estudiantes tienen hacia ellas son favorables, y el posible impacto positivo en el proceso de enseñanza-aprendizaje que dicha implementación podría significar, medido, entre otras cosas, en el rendimiento de las(os)estudiantes, podría también ser positivo. Un próximo estudio podría considerar un análisis comparativo entre un grupo experimental (sección del curso matemáticas 1 o similar en la que se aplique la plataforma Aleks) y un grupo control (sección en la que no se aplique), y cruzar los resultados reportados en el relato con otros indicadores de nivel de aprendizaje como, por ejemplo, rendimiento (lo que llevaría a una investigación mixta). 


\section{Referencias}

Bardin, L. (1991). Análisis de contenido. Madrid, España: Ediciones Akal.

Barraza, A. (2020). El estrés de pandemia COVID 19 en la población mexicana. Centro de Estudios Clínica e Investigación Psicoanalítica S.C. Recuperado de: http://www.upd.edu.mx/PDF/Libros/Coronavirus.pdf.

Bowlby, A. (1988). Secure Base: clinical applications of attachment theory. London: Routledge.

Boykin, K. y Xiao, Z. (2009). New Artificial Intelligence Systems for Improving Student Math Skills: Assessment and Learning in Knowledge Spaces (ALEKS). The National Science Foundation. Recuperado de http://map.ua.edu/ more/EMAP-ALEKS-Distance-Learning.pdf

Breda, A. (2020). Características del análisis didáctico realizado por profesores para justificar la mejora en la enseñanza de las matemáticas. Bolema: Boletim de Educação Matemática, 34(66), 69-88.

Cammaerts, B. (2017). ICT-usage Among Transnational Social Movements in the Networked Society: To Organise, to Mobilise and to Debate. LSE Research Online, 71-90.

Cerasoli, C., Nicklin J. y Ford M. (2014). La motivación intrínseca y los incentivos extrínsecos predicen conjuntamente el rendimiento: un metaanálisis de 40 años. Psychol Bull, 140(4), 980-1008.

Chen, Q., Liang, M., Li, Y., Guo, J., Fei, D., Wang, L., He, L., Sheng, C., Cai, Y., Li, X., Wang, J., y Zhang, Z. (2020). Mental health care for medical staff in China during the COVID-19 outbreak. The Lancet Psychiatry, 7(4), e15-e16.

Cook, D. y Artino, A., (2016), Motivation to learn: an overview of contemporary theories. Med Educ, 50, 997-1014.

Fisher C., Laubascher L. y Brook R. (2016). The Qualitative Vision for Psychology. An invitation to a human science approach. Pittsburgh, Pennsylvania: Duquesne University Press.

Fuster E. (2019). Investigación cualitativa: Método fenomenológico hermenéutico. Propósitos y Representaciones, 7(1), 201-229.

García, C., y Manso, J. (2018). Transforming education for a changing world. Holanda: Adaya Press. 
Gómez, I. y Marbán, J. (2019). Afecto y conocimiento profesional docente en matemáticas. En E. Badillo, N. Climent, C. Fernández, M. González (Ed.) Investigación sobre el profesor de matemáticas: práctica de aula, conocimiento, competencia y desarrollo profesional. (p.397-416). Salamanca, España: Ediciones Universidad de Salamanca.

González, N., Tejada, A., Espinosa, C. y Ontiveros, Z. (2020). Impacto psicológico en estudiantes universitarios mexicanos por confinamiento durante la pandemia por Covid-19. Recuperado de: https://doi.org/10.1590/SciELOPreprints.756

Guerrero-Castañeda, R., Prado, M., Kempfer, S. y Ojeda, M. (2017). Momentos del Proyecto de Investigación Fenomenológica en Enfermería. Index de Enfermería, 26, 67-71.

Guerrero-Castañeda, R., Menezes, T., y Prado, M. (2019). La fenomenología en investigación de enfermería: reflexión en la hermenéutica de Heidegger. Escola Anna Nery, 23. Recuperado de: https://www.scielo.br/j/ean/a/ y6JjfgRX6Q8vkNrrYdSLpWg/?lang=en

Gurung, B. (2015). Pedagogías emergentes en contextos cambiantes: pedagogías en red en la sociedad el conocimiento. Enunciación, 20, pp. 271-286

Heidegger, M. (2015). Ser y tiempo. Santiago de Chile: Trotta.

Hofmann, S. (2018). La emoción en psicoterapia: De la ciencia a la práctica. Barcelona: Paidós.

Inter-Agency Standing Committee (IASC). (2020). Briefing note on addressing mental health and psychosocial aspects of COVID-19 Outbreak, Version 1.1. Recuperado de: http://www.socialserviceworkforce.org/system/files/ resource/files/Briefing-Note-Addressing-Mental-Health-and-PSS-COVID-19.pdf

Jiménez-Domínguez, B. (2000). Investigación cualitativa y psicología social crítica. Contra la lógica binaria y la ilusión de la pureza. Revista Universidad de Guadalajara, 17. Recuperado de: https:/groups.google.com/g/ ic-investigacion-cualitativa/c/IH-fDHxCfdM.

Jin, S. y Cho, C. (2015). Is ICT a New Essential for National Economic Growth in an Information Society?, Government Information Quarterly, 32, 253-260

Khan, S., Siddique, R., Li, H., Ali, A., Shereen, M. A., Bashir, N., y Xue, M. (2020). Impact of coronavirus outbreak on psychological health. Journal of Global Health, 10, 1-6. 
Krippendorff, K. (1990). Metodología de análisis de contenido. Teoría y Práctica. Madrid, España: Paidós Ibérica.

Maćkowski, M., Brzoza, P., Żabka, M. y Spinczyk, D. (2018). Multimedia platform for mathematics interactive learning accessible to blind people. Multimedia Tools and Applications, 77, 6191-6208.

Marín, F. (2018). Actitudes de los estudiantes hacia el estudio de las matemáticas en su entorno familiar y en el aula, un acercamiento desde el dominio afectivo. Acta Latinoamericana de Matemática Educativa, 31, 192-199.

Maurice, M. (2006). Fenomenologia da percepção. São Paulo: WSF Martins Fontes.

Mikulincer, M. y Phillip R.. (2012). An attachment perspective on psychopathology. World Psychiatry, 11, 11-15.

Nwaogu, E. (2012). The effect of ALEKS on students' mathematics achievement in an online learning environment and the cognitive complexity of the initial and final assessments. [Dissertation, Georgia State University]. Recuperado de: https://scholarworks.gsu.edu/msit_diss/94

OEI-IESALC (2020). COVID-19 y Educación Superior: De los efectos inmediatos al día después. Instituto Internacional para la Educación superior en América Latina y el Caribe. Recuperado de: http://www.iesalc.unesco.org/ wp-content/uploads/2020/05/COVID-19-ES-130520.pdf

Organización Mundial de la Salud OMS (2020). Preguntas y respuestas sobre la enfermedad por coronavirus (COVID-19). Recuperado de: https:// www.who.int/es/emergencies/diseases/novel-coronavirus-2019/advicefor-public/q-a-coronaviruses

Ozamiz, N., Dosil, M., Picaza, M. y Idoiaga, N. (2020). Niveles de estrés, ansiedad y depresión en la primera fase del brote del COVID-19 en una muestra recogida en el norte de España. Cadernos de Saúde Pública, 36, 1-9.

Pérez, G. (1998). Investigación cualitativa retos e interrogantes. España: Colofón, S.A.

Pérez D., y Moreno R. (2019). La investigación cualitativa: un camino para interpretar los fenómenos sociales. En J. Mendoza y N. Esparragoza (eds). Educación: aportaciones metodológicas (pp. 85-101). Puebla: Universidad de Oriente.

Quecedo, R. y Castaño C. (2002). Introducción a la metodología de investigación cualitativa. Revista de Psicodidáctica, 14, 5-39 
Quispe, D. (2017). Motivación y hábitos de estudio en estudiantes de la Universidad Alas Peruanas, Filial Juliaca, [Tesis de Maestría] Universidad Andina Néstor Cáceres Velásquez, Perú.

Ramírez, R., Soto, J. y Campos, L. (2020). Motivación educativa y hábitos de estudio en ingresantes de ciencias de la salud. Revista Universidad y Sociedad, 12, 273-279.

Salgado, A. (2007). Investigación cualitativa: diseños, evaluación del rigor metodológico y retos. Liberabit, 13, 71-78.

Sampieri, R. (2018). Metodología de la investigación: las rutas cuantitativas, cualitativa y mix. Ciudad de México: McGraw Hill.

Sánchez, M., García, J., Steffens, E. y Palma, H. (2019). Estrategias pedagógicas en procesos de enseñanza y aprendizaje en la educación superior incluyendo tecnologías de la información y las comunicaciones. Información Tecnológica, 30, 277-286.

Santrock, J. (2019). Adolescence. Minnesota, E.E.U.U.: McGraw-Hill

Scharager, J., Reyes, P. (2001). Muestreo no probabilístico. Metodología de la investigación para las ciencias sociales. Santiago, Chile: Pontificia Universidad Católica de Chile.

Sociedad Interamericana de Psicología Inc. (SIP) (2020). Protocolo de Acción y Recomendaciones sobre Salud Mental para afrontar la Pandemia del Coronavirus. Responsabilidad, Empatía, Solidaridad y Tranquilidad. (Versión 1.1) Recuperado de: https://sipsych.org/wp-content/ uploads/2020/03/SIP-2020-CORONAVIRUS18_03_2020-Español.pdf

Torres, J. y Velandia, S. (2017). Influencia de las Estrategias Pedagógicas en los Procesos de Aprendizaje de los Estudiantes de una Institución de Básica Primaria de la Ciudad de Bucaramanga, Puente, 7, 117-130.

Vasilachis, I. (1992). Métodos cualitativos I. Los problemas teórico-epistemológicos. Buenos Aires, Argentina: Centro Editor de América Latina.

Velázquez, L. (2020). Estrés académico en estudiantes universitarios asociados a la pandemia por COVID-19. Espacio I+D, Innovación Más Desarrollo, 25, 158-179.

Vejar M. y Ávila J. (2020). Emociones de estudiantes de tercer año básico en el contexto de evaluaciones escritas en educación matemática. Paulo Freire. Revista De Pedagogía Crítica, 23, 47-68. 
Wang, C., Pan, R., Wan, X., Tan, Y., Xu, L., Ho, C. S., y Ho, R. C. (2020). Immediate psychological responses and associated factors during the initial stage of the 2019 coronavirus disease (COVID-19) epidemic among the general population in China. International Journal of Environmental Research and Public Health, 17(5). Recuperado de: https://doi.org/10.3390/ ijerph17051729

Recibido: 25.11.2020 Aceptado: 04.03.2021 